\title{
Zang Liver as a Frugality Rhythmic System for Stability for Activities and Against Stress
}

\author{
Edwin Chau-Leung $\mathbf{Y u}^{1}$
}

1 Director, InteMed HK; Honorary Professor, Hong Kong Baptist University (HKBU), Kowloon Tong, Hong Kong

*Corresponding Author: Dr. Edwin Chau-Leung Yu, Director, InteMed HK; Honorary Professor, Hong Kong Baptist University (HKBU), Kowloon Tong, Hong Kong. Email: yuchauleung@gmail.com

Received: April 15, 2019; Accepted: July 11, 2019

\begin{abstract}
The liver reacts sensitively to the body energy state. Metabolic constraints are present when the body diversifies in activities for the many life needs. There is frugality for liver support to metabolic demand in activity. It determines the way of the individual to handle matters of life. Action and perfusion goes together to support metabolism. Circulation to body parts undergoing activities may be raised reactively or anticipatorily by configured patterns from the hepato-splanchnic circulation. Its recruitment from other body parts including the perfusion bed of the gut, the muscles and the brain may cause trade-offs there and need be restored at night. This is one of the mechanisms of autoregulation, akin to dispersion and free-coursing in Zang Liver. Prolonged exhaustion over-drains liver support processes and can lead to perturbations of the dispersion and dredging of the Zang liver. Extraneous activities may cause exaggerated trade-offs in body function including gastrointestinal and mental upsets. Beyond metabolic support by the liver, these may lead to pathologies affected for their metabolism during endeavors. Zang liver dysfunctions similarly arise. Biological terms with the liver as a frugality rhythmic system can help to explain the internal processes producing Zang liver features in Chinese medicine.
\end{abstract}

Keywords: Chinese Medicine, Zang Liver, Frugality rhythmic system.

\section{INTRODUCTION}

Functionally, the five organs in Chinese Medicine (CM) constitute interactively the five vital systems of the body. CM Zang liver is one of them. CM with a different perspective of the human body, diseases and pathology, has a broad view of this liver system as it involves multiple body systems and organs. Currently viewed, among the major functions of the Zang liver in $\mathrm{CM}$, it is in charge of dispersion and dredging, which would be responsible for the regulation of emotion, the promotion of digestion and absorption, the maintenance of the circulation of qi, blood, and body fluid, as well as the reproductive function. It also mediates storing blood and can modulate the blood volume. $\mathrm{CM}$ theory describes that qi and blood become most active in the gallbladder and liver meridians from 11 p.m. to 3 a.m. Short of sleep in this period of time, the liver will fall short of enough nourishment from the blood and cannot perform well in storing blood and keeping hun, hun being the director spirit. By storing blood, the Zang liver supports the normal functions of the eyes, tendons and nails. The Zang liver is also the source of endurance. The described major functions of the CM Zang liver can be explained by current biological terms in this paper.

\section{BASIC ATTRIBUTES OF THE LIVER}

CM Zang liver functions can be appreciated by first understanding the liver's tripartite complex with metabolic, neurologic, and rhythmic signaling control.

\section{A. The liver as a metabolic complex}

The liver as a metabolic organ maintains reserve for replenishing body turnover in energy metabolism. Energy homeostasis essentially balances between storage and usage. Pre- and postprandial mechanisms around the liver keep blood glucose levels constant by glycolysis and gluconeogenesis, with glycogen stored in liver when appropriate.

With the hepatocyte as an important storage compartment, the liver controls through signals to keep the body fuller in reserve or surplus. Thereby, it reacts sensitively to food uptake while interacting with the gut that fills itself from time to time through hunger and satiety [1]. Tightly controlled by metabolic hormones, raised plasma glucose level would lower the subjective appetite and food intake [2]. Inhibition of oxidation of free fatty acid (FA) is also associated with enhanced eating [3]. Glucose and FA metabolism are linked. 
To face unsteady energy fluctuations, particularly so in animal life with uncertainty of food resources, and unceasing need for activity, the body would frugally use or not use the ready stores. Signaling mechanisms start in the liver. A regulator of metabolism, the adenosine monophosphate (AMP)-activated protein kinase (AMPK), coordinates multiple metabolic pathways. It adapts cellular processes to the energy status, by switching from anabolic to catabolic states to re-store energy balance or elevate usage depending when provisions are incremental [4]. Liver kinase B1 (LKB1) phosphorylates and activates AMPK in the liver. This activity is regulated by nutrients and by the cellular energy state in response to a change in the cellular AMP/ATP ratio in physiological processes and pathological stresses [5]. In short, whether liver geared to synthesize or utilize carbohydrate, lipid and protein is tightly regulated according to needs.

\section{B. The liver as a nervous complex}

The liver as a nervous complex is well traversed by innervation down to its parenchymal hepatocytes [6]. Innervations at the portal vein, as a strategic-site sensor after Gl absorption, provides metabolic sensing including osmolality [7], glucose [8] and FA levels [9]. Here, decreased portal glucose concentrations immediately can trigger increased food intake [10] particularly its initiation [11]. It detects free FA levels, affecting feeding behavior [12] and is crucial for directing fat deposition and regulating plasma metabolite levels [13]. The afferent sensory neurons also detect nutritional and absorptive hormones like incretins (that affect insulin release), Gl hormones like somatostatin and leptin $[14,15]$ and cholecystokinin (CCK), which is released in the duodenum in response to food intake.

The liver, Gl tract and brain work together and cater for different needs and inputs for metabolic balances. Through the brain's own metabolic sensors peripherally, and the associated hypothalamic neural mechanisms, glucose metabolism is controlled [16]. A regulatory hypothalamic brain-liver circuit also both decreases liver glucose production [17] and decreases the hepatic secretion of lipids by hepatocytes [18]. Generally, the sympathetic system stimulates whereas the parasympathetic system suppresses hepatic gluconeogenesis [2]. The autonomic system (ANS) also regulates liver FA metabolism, including very low-density lipid [19]. Operationally, the brain and GI tract may act in opposition to the liver [20]. This allows the Gl-liver-brain energy network to act as a mutual-balancing circuit [11].

\section{The liver as for rhythmic control}

The liver is autonomized to face the variable food energy influx, as it expresses a robust circadian pattern. Through transcription factors as regulatory proteins that turn rhythmic genes on-off, hepatocytes affect circadian vital functions, including nitrogen or carbohydrate metabolism, lipid and nutrient homeostasis, bile acid synthesis and metabolism, control of oxidative pathways, cell division, and polyamine synthesis [21,22,23]. The brain suprachiasmatic nuclei (SCN) and the peripheral transcriptome together act as a multi-oscillator system [24].

Matched to external cues, this pacemaker system allows anticipation and preparation in metabolism and activity for environmental changes so as to behave appropriately with right intensity at the right time of day and event. The liver rhythm is synchronized with SCN by direct entrainment through ANS innervation and hormonal signals, but it is not phase-locked to SCN [25]. The liver is significant in the multi-oscillator system since rhythmic feeding is an important entrainment factor [26].
Towards matching diverse internal cues, body operations are configured with patterns. Thus, nutrient and energy metabolism is synchronized for storage and utilization of energy with light/dark cycles $[27,28]$ while hepatic functions (gluconeogenesis, de novo lipogenesis, VLDL secretion, cholesterol biosynthesis, and xenobiotic detoxification) are patterned diversely $[26,29]$. Each of these are precisely circadian-timed to physiological events to reach respective peaks at different times of the day.

The configured rhythms are not set, but adaptive to new life scenarios. Adapting to new environmental changes after some transition time, the innate phasic temporal structure of the oscillators may be re-configured, synchronized to recurring cues, and consolidated after coordination of physiological events. Once activated, these rhythms continue running (sustainment), but the rhythms could become interrupted if the activator disappears. Energy and activity thereby are matched by adjustment between usage and storage and phasematched by anticipatory patterned food-activity cycles. The liver rhythms could be adapted to complex directions and demands as it is tuned to diverse activities including menstruation, work and stress.

\section{FRUGALITY AND RHYTHMS OF THE LIVER IN BODY CONTROL}

Thus, the liver provides the energy reserves for assertive actions. Frugally, knowing the need and storage, the liver controls metabolic provision according to demand. CM Zang liver is similarly described as an "energy capital when body deenergized" [30].

\section{A. Frugality for Energy Support in Activities}

The individual would often come across many issues and need to be involved. It would be easier if unlimited energy were provided for. As this could not be so, this becomes a challenge and the person may become stressed. The frugal support offers reserves for needs that the individual engages, but being frugal, it may not accede support to all sorts of demands, especially futile efforts.

The liver-brain energizer base is evolved in balance and control to cater for environmental and situational demand. Standing strategically at the site of influx of food nutrient and resources, the liver together with the brain maintain metabolic readiness for a vast matrix of metabolic functions and the whole range of body needs. Both reactively and anticipatorily, signaling mechanisms cater for different directions to usage or storage. Also, innately patterned processes match food, light-dark cycles and activities for being efficient and timely with a cost-effective output to meet these diverse needs and activities.

Essentially, the liver phase-coordinates body processes in adaptive patterns to match the environment while facilitating cost savings. For diversified energy-dependent body processes, some of these are precisely timed to reach their respective peaks at different times. Besides, the liver functioning with rhythmic patterns as a tripartite complex became a significant controller unit. Assertive actions need metabolic support. Metabolism needs perfusion. The liver also supports circulatory redistribution. 


\section{B. Recruiting Circulatory Support for Activity.}

\section{Reactive Redistribution}

Energy and perfusion are coupled to support action. Beyond reserves for basic sufficiency, any enhanced activity requires extra perfusion, and blood flow for that part of the body. This has to be recruited from other parts, with trade-offs. In particular, during peak effort, skeletal muscle blood flow is about $90 \%$ of peak cardiac output, some 18 -fold increase from that about $20 \%$ at rest. Since increased cardiac output and stored body fuels cannot meet this sufficiently, this peak demands about $10 \%$ of the total flow to muscle be borrowed from other areas (skin, gut) where reoxygenation can be postponed. Circulatory recruitment from other body parts including the perfusion bed of the gut, the muscles and the brain may cause tradeoffs there and need be restored at night. The body goes into restorative processes afterwards, particularly during sleep time when energy consumption is minimal.

Histologically, the liver is a blood reservoir that consists of hepatic sinusoids, which should be the histological foundation of the Zang liver in storing blood [31]. Mediated through sympathetic stimulation, blood from the hepatic large capacitance vessels including the sinusoids, and from the even larger capacitance of the splanchnic system may be recruited when stressed. In a low-flow state, splanchnic blood flow decreases in order to maintain vital cardiac and cerebral blood supply [32], and may mobilize up to $5-6 \%$ of total blood volume in response to physiologic challenges.

\section{Blood Redistribution in Anticipation for activities}

This reactive mode by recruitment with trade-offs and restoration afterwards could produce metabolic lag times and generation of wastes. The body has another efficient way to handle needs for activity anticipatorily. Most of the daily activities are patterned, and with the multiple pacemakers, metabolism and perfusion are patterned along with these activities. Internal oscillators exist in endothelial, smooth muscle cells, and fibroblasts of the vasculature. Vascular tone and thrombus formation, two key elements of vascular, exhibit diurnal rhythmicity [33]. The body circadian clock regulates many other aspects of activity physiology including cardiovascular function. These can be patterned according to activity patterns.

These patterned regulatory processes form an important mechanism underlying the $\mathrm{CM}$ dispersion and dredging function. $\mathrm{CM}$ describes its capacity for regulation of snug body dynamics ("free coursing") by redistributing energy and perfusion, maintaining blood vessels open and unobstructed, regulating gastrointestinal transport, modulating emotional flows and promoting reproductive mechanisms. The dispersion function should actually be properly ascribed to these auto-regulatory processes driven by the hepato-splanchnic circulation and liver patterned dynamics. Studies suggest that the liver dispersion is related to the neuroendocrine- immune network [34]. When the liver processes are patterned well, activities in health will be snug in good timing. In congruence and snug, one can work well and respond readily to diversified operations for activities.

\section{POWER TO FACE STRESS}

It is during times of over-diversifying activities unmatched by frugality provisions and by innate or configured patterns for the activity that stress occurs. Both metabolic and perfusion needs are lagging in timeliness and strained in these situations. The auto-regulatory processes supported by the hepato-splanchnic circulation and liver pattern lose their snug dynamics so as to affect gastro-intestinal function and mental support for smooth and snug living.

However, supported, metabolic constraints are certainly present. Energy provision could not be of unlimited supply to ever demanding diversifying endeavors. An active brain part may be so highly perfused and metabolizing that requires extra blood perfusion recruited from the other brain regions within the brain itself [35].

When the body at night returns to the unperturbed basic natural rhythm with no more extraneous activity and no more elevations, and when eyes closed with no more dissipative involvement, hepato-splanchnic circulation trade-offs are restored, reviving integrality and entirety. The output of the brain neurohumoral system would be different depending upon the sleep state. Sleep of sufficient duration, continuity, and depth without circadian disruption is necessary to promote high levels of attention and cognitive performance during the wake period.

Beyond metabolic stability enabled by the liver base, extra demanding functions during diversifying activities call for extra body drives. Coordinative processes in the physical body frame would become stressed, and the autonomous processes thrown out of equilibrium. With demanding circumstances and challenges, internal metabolic and neural facilitative mechanisms activate glucosteroids (GC) and the stressresponsive hypothalamic-pituitary axis (HPA). GC is key to synchronicity of SCN and liver circadian time configuration [36], and important to the rhythmic expression of a large number of livers genes [37]. Ordinarily, cortisol peaks in the morning to boost up physical and mental performance [38]. Rhythms in neurohormonal patterns including GC boost up the phase coordination. Thereby, the CM Zang liver dispersion and dredging function would be similarly enhanced.

Understanding that the provisions to sustain proficiency are frugal would explain exhaustion problems. Nature tends towards escalating rather than being subdued. Diversifying issues continue to come in. With the given or allowed provisions, how to contain the costs for the demand and how to embrace the size of projects could shape the nature of mind and body coordination. In volatile and unpredictable environment, there could be no ways to buildup reliable mechanisms to match with cost and capabilities for living. For the prepared (or overprepared), a person may per adventure have escalated from his portfolio of patterned and prepared packages of energy resources developed for tackling processes. Prepared for a feat, the lock-in mental and physical processes would be installed beforehand for that matter. Giving up the involved area of activity when it is found not cost-effective for living, would mean dropping out the prepared packages. Pent-up processed energy and prepared resources unreleased could only pass out by dematerialization even by apoptosis internally or maybe by rage externally. In studies on Rage Damaging Zang Liver, decreased intracellular protein and central lobular cell apoptosis were noted [39], analogous to clearing out messy budgets from sunken costs. The liver with high capacity to regenerate, has apoptosis as a normal mechanism Liver regeneration is circadian regulated [40]. Polyamines, regulated by the hepatic circadian clock [23], are highly associated with cell proliferation and are essential for the initiation of liver regeneration. Recent evidence indicates that AMPK is involved in autophagy in hepatocyte, revealing a direct connection between energy status and autophagy initiation [41]. Liver cell autophagy is critical for cellular homeostasis and nutrient metabolism [42]. In parallel, 
autophagy is required for removing protein aggregates, damaged organelles, and certain pathogens [43]. The coupling of autophagy and metabolism is more and more understood as a factor underlying metabolic homeostasis and disease.

In volatile environment straining the body, the individual could be equipped with many fixed tackling processes to finishing tasks. But as the life course gets varied, the many futile processes mean toiling the body and wasting much energy resources. This is especially so when this is a prolonged attempt to grudge or fight against possible offensive situations. In animal models of Liver Suppression, the hepatocytes may be said to be exhausted with demonstrable cell pathology [44]. This pathology is often seen when tackling processes for situations meet too much discrepancy.

Hardships are often related to feelings cumulated for the lack of proficient support during the processes. Cumulated long-term tradeoffs may be overdue. Over-stressed, in time, behavioral responses adapting and adjusting to environmental events may have conflicting motivations in the context of future needs and resource allocation. These have effects on emotions.

\section{CONCLUSION}

The liver's metabolic resourcefulness is important in the balance and control of energy homeostasis for supporting activity. The Zang liver is the capital when body de-energized, generating blood and qi processes" [30] so that it is the source of endurance. Starting from gut resources, the pattern-configurable liver-brain base supports the mental and physical dynamics and the associated reactive and patterned recruitment of hepatosplanchnic circulation. CM Zang liver that mediates storing blood and modulates blood volume is one important feature for its dispersion and dredging function. The hypothalamus and ANS are also closely involved in the drive for signaling and pattern for the process of regulation of redistribution. When the coordinative processes become stressed and the autonomous processes thrown out of equilibrium, the dispersion function is needed and this could be ascribed to auto-regulatory processes. Gaps may activate GC and the stress-responsive HPA. The frugality of liver support for metabolic demand in activity determines the way of the individual to handle matters of life and may be expressed in emotions and pathological liver changes in rage or Zang Liver Suppression.

The liver reacts sensitively to the body energy state. When working or excited, the liver releases the stored blood to meet the requirements of the body and to maintain normal activity function. Trade-offs are restored at night. CM similarly described the Zang liver stores blood, and when the body moves, blood circulates in the channels, while at rest, blood returns to the liver. The frugal mode and rhythmic patterns determine the liver's provision in matching activities. Overly assertive use of supportive resources for diversifying endeavors creates gaps. $\mathrm{CM}$ described the liver-blood often inadequate. When the body functions in unperturbed basic natural rhythm with no more extraneous activity and no wilder elevated demanding activities, the Zang liver is harmonious and the body snug.

As a whole, attempts in the past to describe Zang liver and its basic manifesting functions (Zang Xiang features) based around the actual liver organ itself have found difficulties. The main difficulty could be due to CM workers following the study of Western medicine depicts the liver as just a metabolic organ. In this paper, perceiving the liver as it should be, as a controlling organ helps reconstructing pathophysiology. The biological basis for this is outlined. Particularly, how the liver functions to frugally maintain body metabolism, and the configurable pattern body processes are emphasized. These help to understand the effects on the hepatosplanchnic autoregulation and effect on body snug process analogous to dispersion mechanisms of the Zang liver. With resources synchronized, the better match the determined activity diversification to environment, the better the body strung in mastery. Else, upsetting core snugness needs autoregulatory dispersion and dredging.

The liver is one closely interactive inter-coordinated complex with the gut, brain and hepatosplanchnic circulation. It helps the person to face the environment with coordinative processes cost-effectively as energy homeostasis is kept up and maximized by recruitable gastrointestinal (Gl) and circulatory reserves, allowing for restoration afterwards. Biological terms with the liver as a frugality rhythmic system can help to explain Zang liver functions.

\section{REFERENCES}

1. Dufour JF, Clavien PA (ed). Hepatocytes in Signaling Pathways in Liver Diseases 3/e, Chpt 1, John Wiley \& Sons Ltd, 2015.

2. Rui L. Energy Metabolism in the Liver. Compr. Physiol., 4(1): 177197, 2014.

3. Langhans W. Role of the liver in the control of glucose-lipid utilization and body weight. Curr. Opin. Clin. Nutr. Metab. Care, 6(4): 449-55, 2003.

4. Foretz $\mathrm{M}$ and Viollet $\mathrm{B}$. Regulation of hepatic metabolism by AMPK. J.Hepatol., 54(4):827-9, 2011.

5. Patel K, Marion A, Campbell DG, Gourlay R, Boudaba N, Tournier $E$, et al. The LKB1-salt-inducible kinase pathway functions as a key gluconeogenic suppressor in the liver. Nature Communications, 2014.

6. Fukuda $\mathrm{Y}$, Imoto M, Koyama $\mathrm{Y}$, Miyazawa $\mathrm{Y}$, Hayakawa $\mathrm{T}$. Demonstration of noradrenaline-immunoreactive nerve fibres in the liver. J. Int. Med. Res., 24:466-472, 1996.

7. Abe $\mathrm{CH}$. Negative feedforward control of body fluid homeostasis by hepatorenal reflex. Hypertens. Res., 34:895-905, 2011.

8. Hevener AL, Bergman RN, Donovan CM. Hypoglycemic detection does not occur in the hepatic artery or liver: findings consistent with a portal vein glucosensor locus. Diabetes, 50:399-403, 2001.

9. Randich A, Spraggins DS, Cox JE, Meller ST, Kelm GR. Jejunal or portal vein infusions of lipids increase hepatic vagal afferent activity. Neuroreport, 12:3101-3105, 2001.

10. Niijima A. The effect of D-glucose on the firing rate of glucosesensitive vagal afferents in the liver in comparison with the effect of 2-deoxy-D-glucose. J Auton. Nerv. Syst., 10:255-260, 1984.

11. Yi CX, la Fleur SE, Fliers E, Kalsbeek $A$. The role of the autonomic nervous liver innervation in the control of energy metabolism. Biochim. Biophys. Acta., 1802:416- 431, 2010.

12. Cox JE, Kelm GR, Meller ST, Spraggins DS, Randich A. Truncal and hepatic vagotomy reduce suppression of feeding by jejunal lipid infusions. Physiol. Behav., 81:29-36, 2004.

13. Warne JP, Foster MT, Horneman HF, Pecoraro NC, Ginsberg AB, Akana SF, Dallman MF. Afferent signalling through the common hepatic branch of the vagus inhibits voluntary lard intake and modifies plasma metabolite levels in rats. J.Physiol., 583:455467, 2007.

14. Nakabayashi H. Neural monitoring system for circulating somatostatin in the hepatoportal area. Nutrition, 13:225-229, 1997.

15. Shiraishi T, Sasaki K, Niijima A, Oomura Y. Leptin effects on feeding-related hypothalamic and peripheral neuronal activities in normal and obese rats. Nutrition, 15:576-579, 1999.

16. Watts AG, Donovan CM. Sweet talk in the brain: glucosensing, neural networks, and hypoglycemic counterregulation. Front 
Neuroendocrinol., 31:32-43, 2010.

17. Pocai A, Obici S, Schwartz GJ, Rossetti L. A brain-liver circuit regulates glucose homeostasis. Cell Metab., 1:53-61, 2005.

18. Jensen KJ, Alpini G, Glaser S. Hepatic Nervous System and Neurobiology of the Liver. Compr. Physiol., 3(2): 655-665, 2013.

19. Yamauchi T, Iwai M, Kobayashi N, Shimazu T. Noradrenaline and ATP decrease the secretion of triglyceride and apoprotein B from perfused rat liver. Pflugers Arch., 435:368-374, 1998.

20. Caspi L, Wang PY, Lam TK. A balance of lipid-sensing mechanisms in the brain and liver. Cell Metab., 6:99-104, 2007.

21. Jessica M. Ferrell and John Y.L. Chiang. Circadian rhythms in liver metabolism and disease. Acta. Pharm. Sin. B., 5(2): 113-122, 2015.

22. Reddy AB, Karp NA, Maywood ES, Sage EA, Deery M, O'Neill JS, Wong GK, Chesham J, Odell M, Lilley KS, et al. Circadian orchestration of the hepatic proteome. Current Biology, 16:11071115, 2006.

23. Atwood A, DeConde R, Wang SS, Mockler TC, Sabir JS, Ideker T, Kay SA. Cellautonomous circadian clock of hepatocytes drives rhythms in transcription and polyamine synthesis. Proc. Natl. Acad. Sci. USA., 108:18560-5, 2011.

24. Miller $\mathrm{BH}$. Circadian and CLOCK-controlled regulation of the mouse transcriptome and cell proliferation. Proc. Natl. Acad. Sci. USA., 104(9): 3342-7, 2007.

25. Schmutz I, Albrecht U, Ripperger JA. The role of clock genes and rhythmicity in the liver. Molecular and Cellular Endocrinology, 349:38-44, 2012.

26. Kornmann B, Schaad O, Bujard H, Takahashi JS, Schibler U. System-driven and oscillator-dependent circadian transcription in mice with a conditionally active liver clock. PLoS. Biol., 5(2): e34, 2007.

27. Green CB, Takahashi JS, Bass J. The meter of metabolism. Cell, 134: 728-742, 2018.

28. Asher G, Schibler U. Crosstalk between components of circadian and metabolic cycles in mammals. Cell Metab., 13: 125-137, 2011.

29. Storch KF, Lipan O, Leykin I, Viswanathan N, Davis FC, Wong $\mathrm{WH}$, Weitz CJ. Extensive and divergent circadian gene expression in liver and heart. Nature, 417(6884): 78-83, 2002.

30. (唐)王冰, 黄帝内经. 素问, 第三卷: 第九篇, 六节藏象论。

31. He FJ, Wang J, Niu JZ: Study progress on hepatic sinusoidal wall cells. Jie Pou Ke Xue Jin Zhan 2001; 7: 156-157.

32. Jakob SM, Tenhunen JJ, Laitinen S, Heino A, Alhava E, Takala J. Effects of systemic arterial hypoperfusion on splanchnic hemodynamics and hepatic arterial buffer response in pigs. Am. J. Physiol. Gastrointest. Liver Physiol., 280:G819-G827, 2001.

33. Georgios K. Paschos and Garret A. FitzGerald. Circadian Clocks and Vascular Function. http://dx.doi.org/10.1161/CIRCRESAHA.109.211706 Circulation Research. 2010;106:833841.

34. Chen JX, Yang WY: Exploration on research situation of nervesendocrineimmunologic network and its relationship with liver of Chinese medicine. Beijing Zhong Yi Yao Da Xue Xue Bao 18: $7-$ 11, 1995.

35. Lennie P. The cost of cortical computation. Curr. Biol., 13:493-7, 2003.

36. Balsalobre A, Brown S A, Marcacci L, Tronche F, Kellendonk C, Reichardt H M, Schutz G, U. Schibler.Resetting of circadian time in peripheral tissues by glucocorticoid signaling. Science, 289: 2344-2347, 2000.

37. Reddy AB, Maywood ES, Karp NA, King VM, Inoue Y, Gonzalez FJ, Lilley KS, Kyriacou CP, Hastings MH. Glucocorticoid signaling synchronizes the liver circadian transcriptome. Hepatology, 45, 1478-1488, 2007.

38. Dijckmans B, Tortosa-Martínez J, Caus N, González-Caballero G, Martínez-Pelegrin B, Manchado-Lopez C, Cortell-Tormo J M, Chulvi-Medrano, and Clow A. Does the diurnal cycle of cortisol explain the relationship between physical performance and cognitive function in older adults? Eur. Rev. Aging Phys. Act., 14: 6, 2017.

39. 王朝勋、李玉华, 怒伤肝与神经-内分泌-免疫系统失调探析。辽宁 中医杂志。 $1997 ; 5: 205-206$ 。

40. Matsuo T, Yamaguchi S, Mitsui S, Emi A, Shimoda F, Okamura $\mathrm{H}$. Control mechanism of the circadian clock for timing of cell division in vivo. Science. 302:255-9, 2003.

41. Meijer AJ, Codogno P. AMP-activated protein kinase and autophagy. Autophagy, 3:238-240, 2007.

42. He C, Klionsky DJ. Regulation mechanisms and signaling pathways of autophagy. Annu. Rev. Genet., 43: 67-93, 2009.

43. Mizushima N, Komatsu M. Autophagy: renovation of cells and tissues. Cell, 147: 728-741, 2011.

44. 吕志平、刘承才, 肝郁动物模型肝细胞线粒体超微结构观察。湖 南中医杂志。2000; 16(9) : 61。 